\title{
ARTIGOS
}

\section{ABORDAGENS METODOLÓGICAS DAS PESQUISAS EM ORGANIZAÇÃO E REPRESENTAÇÃO DO CONHECIMENTO NO CONTEXTO BRASILEIRO}

\author{
METHODOLOGICAL APPROACHES OF THE RESEARCHES IN \\ KNOWLEDGE ORGANIZATION AND REPRESENTATION IN BRAZILIAN CONTEXT
}

${ }^{1}$ Camila Monteiro de Barros

2Luciane Paula Vital

Universidade Federal de Santa Catarina ${ }^{1,2}$

\section{Correspondência}

Camila Monteiro de Barros

Universidade Federal de Santa Catarina

Florianópolis, Santa Catarina - Brasil.

E-mail: camila.c.m.b@ufsc.br

Submetido em: 22/10/2018

Aceito em: 09/04/2019

Publicado em: 10/05/2019

Checagem Antiplagiarismo

\section{turnitind}

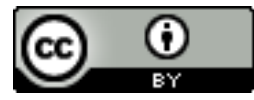

JITA: ID. Knowledge representation.

e-Location ID: 019018 


\section{RPEO $\begin{aligned} & \text { Revista Digital Biblioteconomia e Ciência } \\ & \text { da Informação }\end{aligned}$ PPBOI $\begin{aligned} & \text { Digital Journal of Library and Information } \\ & \text { Science }\end{aligned}$}

RESUMO

O conhecimento científico é caracterizado como racional, sistemático e verificável, sua verificação é viabilizada por meio de metodologias científicas. A construção do conhecimento científico requer métodos que forneçam um aporte adequado às características das diferentes áreas. Esse estudo direciona sua análise para a Organização do Conhecimento, que evidencia-se como nuclear na Ciência da Informação pois, dito de forma simplista, viabiliza a organização da informação por meio da explicitação de conceitos e suas relações, de forma contextualizada, possibilitando a recuperação. Apresenta como objetivo conhecer os enfoques metodológicos utilizados pelos pesquisadores da área de Organização do Conhecimento no Brasil. A pesquisa é de natureza quali-quantitativa, caracteriza-se como exploratória e utiliza como procedimento metodológico a pesquisa bibliográfica. O corpus é composto por 184 trabalhos apresentados no Grupo de Trabalho 2 do Encontro Nacional de Pesquisa em Ciência da Informação (ENANCIB) entre os anos 2013 e 2017. Observa-se uma predominância de trabalhos com abordagem qualitativa (90,2\%), o que condiz com a natureza do objeto estudado: o conceito. É possível verificar que a maior parte das pesquisas (76\%) é teórica, ou seja, verificam, analisam e/ou constroem teorias. 82 trabalhos analisados apresentam mais de um procedimento metodológico, e desses, 4 apresentam mais que 2 procedimentos metodológicos. Verifica-se que é recorrente a ausência de indicação clara dos procedimentos metodológicos adotados, fragilizando a validação das pesquisas. Conclui que as pesquisas em Organização do Conhecimento, no contexto brasileiro, desenvolvem pesquisas com abordagens qualitativas, teóricas e que são fomentadas pela própria literatura científica.

\section{PALAVRAS-CHAVE}

Organização do conhecimento. Ciência da informação. Método de pesquisa.

\section{ABSTRACT}

Scientific knowledge is characterized as rational, systematic and verifiable, its verification is made possible through scientific methodologies. The construction of scientific knowledge requires the adoption of appropriate methods according to the characteristics of the different areas. This study focused its analysis on Knowledge Organization field, which is nuclear in Information Science, as in a simplistic way, it makes possible information organization through concepts explanation and their relations in a contextualized way, improving information retrieval. The main objective was to know the methodological approaches used by the researchers in Knowledge Organization area. The research is characterized as exploratory, with a qualitative-quantitative approach, and uses the bibliographic research as methodological procedure. The corpus was composed of 184 papers presented in Working Group 2 of the Encontro Nacional de Pesquisa em Ciência da Informação (ENANCIB), between 2013 and 2017. It was observed a predominance of woks with a qualitative approach (90.2\%), which seems to correspond to the nature of the object studied: the concept. It was possible to verify that most researches (76\%) are theoretical, that is, they verify, analyze and construct theories. Of the 184 papers analyzed, 82 presented more than one methodological procedure, of which 4 presented more than 2 methodological procedures. It is verified that it is recurrent the lack of clear indication of the adopted methodological procedures, weakening the validation of the researches. It concludes that the researches in Organization of the Knowledge, in the Brazilian context, develop researches with qualitative, theoretical approaches and that are fomented by the own scientific literature.

\section{KEYWORDS}

Knowledge organization. Information sciences. Scientific methods. 


\section{RPBOI $\begin{aligned} & \text { Revista Digital Biblioteconomia e Ciência } \\ & \text { da Informação }\end{aligned}$ RPBOI Digital Journal of Library and Information

\section{Introdução}

O conhecimento científico é caracterizado como racional, sistemático e verificável, sua verificação é viabilizada por meio de metodologias científicas. A construção do conhecimento científico requer métodos que forneçam um aporte adequado às características das diferentes áreas. A Ciência da Informação (CI) foi apontada por Wersig (1993) como um protótipo de uma nova ciência, que necessita de métodos diferentes da ciência chamada 'tradicional'. A definição de uma "nova ciência” encontra solo fértil para discussões na área, suscitando conhecidas indagações como: Seria a CI um novo nome para antigas práticas? Essa "nova ciência” apresenta quais características que a colocam nesse patamar? A resposta a essas questões não é objetivo dessa pesquisa, aqui nos interessa a caracterização da CI como uma ciência que faz uso de diferentes métodos científicos para abordar seus objetos e práticas. Saldanha (2010, p.303) afirma que,

[...] pensar o campo (hoje consagrado com, no mínimo, duas expressões Informacion Science e Library and Information Science) é pensar não em um objeto, mas em uma prática: organizar - conferir um arranjo a um conjunto de manifestações simbólicas ou materiais visando sua resistência no tempo (permanência) e seu acesso no espaço (liberdade).

Mais especificamente, esse estudo direciona sua análise para os métodos de pesquisa adotados na área de Organização do Conhecimento (OC), que se evidencia como nuclear na CI. Dito de forma simplista, e complementando a citação de Saldanha (2010), a OC viabiliza a organização da informação por meio da explicitação de conceitos e suas relações, de forma contextualizada, possibilitando a recuperação. De acordo com Sales (2015) não há consenso no que diz respeito à relação entre CI e OC. O autor afirma que em certas tradições essas são vistas como áreas independentes, vinculadas somente por certos interesses de pesquisas. Em outras tradições, o autor afirma que a OC é vista como uma parte da CI.

A Associação Nacional de Pesquisa e Pós-Graduação em Ciência da Informação (ANCIB) promove o Encontro Nacional de Pesquisa em Ciência da Informação (ENANCIB), que é organizado em 11 Grupos de Trabalho (GT’s). Um desses GT’s é específico para discutir as questões pertinentes à Organização e Representação do Conhecimento, GT2, que se constitui em um importante espaço de socialização das pesquisas desenvolvidas em torno dessa temática no Brasil. Nesse contexto, e considerando que a Organização do Conhecimento tem como preocupações caracterizar e ordenar o conhecimento (SMIRAGLIA, 2013), quais seriam os 


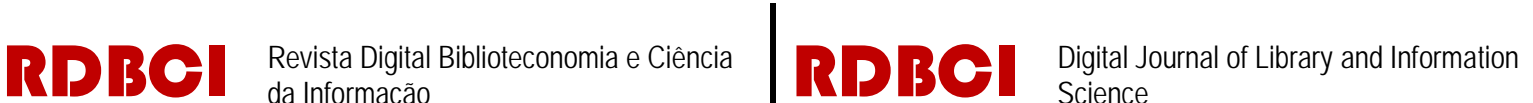

procedimentos metodológicos estão sendo adotados nas pesquisas desenvolvidas nessa área no Brasil?

Assim, essa pesquisa objetiva conhecer os enfoques metodológicos utilizados pelos pesquisadores da área de Organização e Representação do Conhecimento, que publicam no Grupo de Trabalho 2 do ENANCIB (GT 2 ENANCIB). Justifica-se pelo fato de que, evidenciando e problematizando as abordagens metodológicas utilizadas pelo GT, é possível amadurecer e avançar no processo de consolidação das pesquisas na área e fomentar a discussão acerca da institucionalização da Organização do Conhecimento no país.

\section{Perspectivas Epistemológicas em Organização do Conhecimento}

A epistemologia de uma área de conhecimento é delineada pelas teorias, princípios, objetos, objetivos e hipóteses que constituem sua gênese e desenvolvimento (JAPIASSÚ; MARCONDES, 2006). De acordo com Hjorland (1998), a Organização do Conhecimento (OC) apresenta quatro perspectivas epistemológicas centrais: empirismo, racionalismo, historicismo e pragmatismo. Para Smiraglia (2013, p. 2, tradução nossa), do ponto de vista do empirismo, "o conhecimento abrange o que podemos observar". Já do ponto de vista do racionalismo, "o conhecimento abrange o que podemos discernir logicamente", isto é, envolve abstração que pode se aproximar mais ou menos do idealismo. O historicismo, de acordo com Smiraglia (2013), analisa o conhecimento a partir da experiência passada, por meio de uma perspectiva cultural, e por fim, no pragmatismo, o conhecimento é contextual, sendo validado em um determinado período temporal. As origens das concepções empirista e racionalista têm relação, respectivamente, com as ciências naturais e com a lógica e matemática (SMIRAGLIA, 2013).

Para Saldanha (2010) as abordagens que mantém a ligação com as ciências naturais aparecem na OC como pesquisas que buscam "a verdade”, geralmente repousando em uma filosofia representacionista e buscando uma linguagem essencial generalizável. O autor exemplifica sua afirmação com os estudos bibliométricos, automação de processos de indexação, entre outros. Ainda assim, há que se considerar a relevância desses tipos de pesquisa, que respondem certas demandas de soluções em tratamento e acesso à informação.

As concepções historicista e pragmática têm aparecido, de forma crescente, nos estudos de organização do conhecimento. Weiss e Bräscher (2014) sintetizam, com base na literatura, as características da abordagem pragmática na OC: informação é construída, ocorre em contextos múltiplos e heterogêneos e sua qualidade não é constante; a prática por meio da interação é importante para a construção e validação do significado ligado à informação; a bibliografia é uma fonte para 


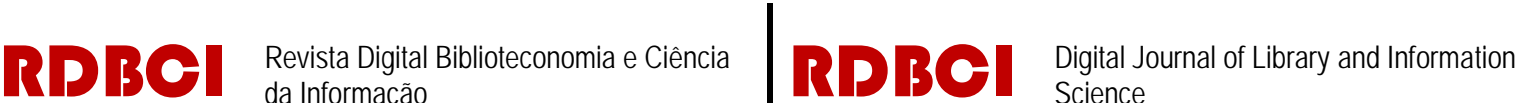

identificar pontos de vista distintos e construir linguagens de comunicação; guiar o usuário na recuperação da informação que vá além daquilo que já é esperado, ampliando escolhas.

Por um lado, os desafios impostos pela ideia de heterogeneidade e dinamicidade do conhecimento - objeto de estudo da OC - pode fazer com que a discussão dessas ideias paire no âmbito teórico e demorem mais tempo para se converterem em aproximações metodológicas. Por outro lado, o historicismo e a pragmática vêm contribuindo nos estudos da OC para o acolhimento das variações contextuais dos significados que compõem o conhecimento.

Mesmo dentro de certa perspectiva epistemológica, que se configura como uma visão mais macro de uma área de conhecimento, é possível explorar essa mesma área com base em uma visão mais micro, que se debruça na individualidade de cada pesquisa desenvolvida. Essa visão micro pode se valer da perspectiva dos procedimentos metodológicos elencados para a realização das pesquisas, ou seja, do conjunto de processos lógicos seguidos quando da delimitação da abordagem, coleta, processamento, análise de dados, etc. (LAKATOS; MARCONI, 2010).

Em alguns casos, tais processos lógicos se limitam à exposição de críticas e reflexões, sem apresentar sistematização metodológica, como é o caso do ensaio. De acordo com Larrosa (2003), esse modo de escrita tem a subjetividade como característica determinante e, portanto, se afasta da isenção e objetividade geralmente buscadas na escrita acadêmica. Para o autor, a produção do ensaio permite que o pesquisador exerça também seu papel de leitor, já que tem certa liberdade na construção do seu pensamento e na escolha dos pontos teóricos a expor.

Valentim (2005, p.19) afirma que "Os diferentes tipos e enfoques de pesquisa estão relacionados à forma de construção de uma determinada área do conhecimento, estão relacionados também à prática de pesquisa de uma determinada comunidade científica.” A escolha dos métodos de pesquisa, ou seja, do método de aproximação e análise do objeto a ser estudado, impacta na definição de quais dimensões desse objeto serão atingidas (hipóteses) e reveladas (objetivos) e, consequentemente, contribuem também de forma relevante para o delineamento epistemológico da área.

Entretanto, classificar o método de pesquisa empregado em determinado estudo não é tarefa fácil. Sabe-se que, apesar do rigor próprio da pesquisa científica, certas nuances subjetivas que podem figurar no momento da coleta e/ou análise dos dados nem sempre são passíveis de serem descritas com precisão. Além disso, a literatura apresenta distintos pontos de vista com relação à descrição e nomeação do método, resultando em diferentes tipos de classificação, como por exemplo: qualitativo, quantitativo e misto (CRESWELL, 2007); métodos de pesquisa qualitativa com enfoque na área da Ciência da Informação (CI): análise

\begin{tabular}{l|l|l|l|l|l|}
\hline C RDBCl: Rev. Digit. Bibliotecon. Cienc. Inf. & Campinas, SP & v.17 & $1-16$ & e019018 & 2019 \\
\hline
\end{tabular}




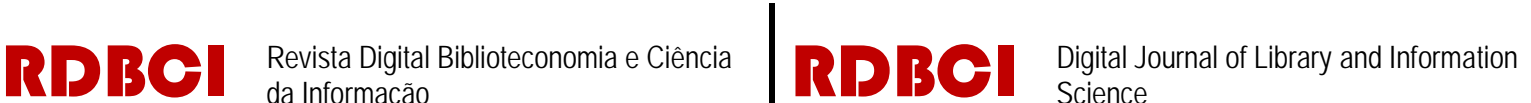

de redes sociais, grupos de foco, análise de conteúdo, etc. (VALENTIM, 2005); classificação de pesquisas de acordo com sua finalidade (básica, aplicada) ou objetivos (exploratória, descritiva, explicativa) (GIL, 2010); técnicas de pesquisa como pesquisa documental, bibliográfica, entrevista, observação, estudo de caso (LAKATOS; MARCONI, 2010); entre muitos outros. Há que se considerar também o interesse do autor em detalhar de forma mais ou menos precisa certos aspectos do método adotado, de acordo com o que lhe parece relevante frente ao seu objeto de estudo.

Considerando esses aspectos, a construção do panorama dos métodos adotados na OC pode incorrer no equívoco da generalização demasiada, deixando de lado a riqueza que a variedade de abordagens metodológicas pode vir a representar. Frente a tal desafio, adotamos na presente pesquisa o viés indutivo, ou seja, a classificação dos métodos de pesquisa ocorreu na medida em que o corpus foi se apresentando, revelando um caráter plural de abordagens, como se verá nos resultados.

\section{Procedimentos Metodológicos}

A pesquisa é de natureza quali-quantitativa, caracteriza-se como bibliográfica e exploratória, utiliza como técnica a pesquisa bibliográfica. O GT2 - Organização e Representação do Conhecimento - do ENANCIB foi escolhido como corpus da pesquisa por se constituir em um dos principais fóruns de debates da Ciência da Informação no Brasil, área em que a OC encontrou espaço de discussão, especialmente nos cursos de pós-graduação. O corpus é composto por 184 trabalhos apresentados no GT2, de 2013 a 2017, disponibilizados na base Benancib, contemplando 5 anos de levantamento, que configura amostragem suficientemente representativa.

Os resumos dos trabalhos foram analisados e classificados de acordo com o quadro 1. Nos casos em que o resumo não apresentava a caracterização do procedimento metodológico da pesquisa, procedeu-se a análise do texto na íntegra.

As categorias apresentadas na primeira coluna do quadro 1 foram definidas a priori; os tipos de pesquisa apresentados na segunda coluna foram levantados de forma indutiva. Para fins de contagem, os métodos que apresentavam similaridades foram agrupados sob o mesmo termo, por exemplo, "levantamento bibliográfico" e "pesquisa bibliográfica”. Alguns trabalhos não declararam o método utilizado, mas, ainda que de forma subjetiva, descreviam certos procedimentos. Nesses casos, os métodos foram nomeados arbitrariamente, entretanto, foram assinalados como "método não declarado". Utilizou-se também a categoria “Outros” para indicar procedimentos metodológicos que não foram nomeados pelos autores. 


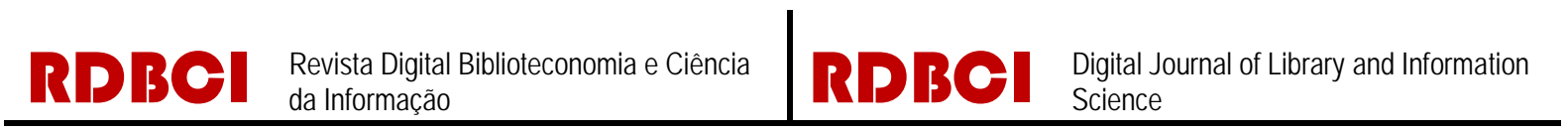


Quadro 1- Categorias de análise

\begin{tabular}{|c|c|}
\hline CATEGORIAS & TIPOS \\
\hline NATUREZA & Teórica \\
Aplicada \\
Outro
\end{tabular}

Fonte: Dos autores (2018)

Para cada artigo analisado foram retirados extratos que ilustram a indicação do(s) procedimento(s) metodológico(s) adotado(s), e alguns serão apresentados nos resultados.

\section{Resultados}

Os resultados serão apresentados de acordo com as categorias analisadas. O gráfico 1 apresenta o número de trabalhos distribuídos pelos anos analisados, sendo possível verificar uma oscilação nessa distribuição. 


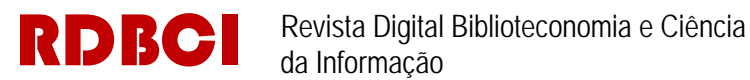

Gráfico 1: Número de trabalhos publicados por ano.

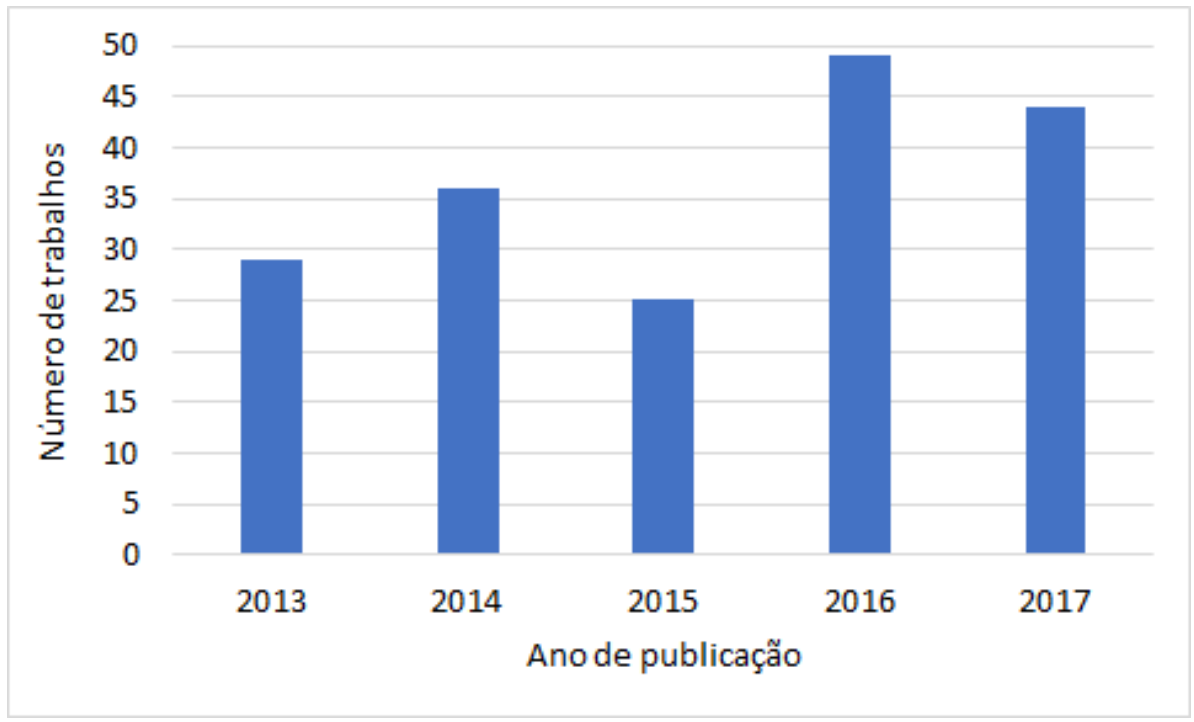

Fonte: Dos autores (2018).

O ano de 2015 é o que tem o menor número de trabalhos apresentados no GT 2 do ENANCIB, no período analisado. Apesar de não ser objetivo desta pesquisa, podem-se levantar algumas hipóteses para a inconstância no número de trabalhos, como a região na qual o evento é realizado. Nos dois anos com menor número de trabalhos, 2013 e 2015, o evento ocorreu em regiões extremas do Brasil, Florianópolis/SC e João Pessoa/PB, respectivamente, tornando mais onerosa a participação.

O gráfico 2 mostra as abordagens das pesquisas, classificadas em qualitativa, quantitativa e quali-quantitativa. Observa-se uma predominância de trabalhos com abordagem qualitativa (90,2\%), o que condiz com a natureza dos objetos estudados, como informação e conhecimento. 
Gráfico 2: Abordagem da pesquisa.

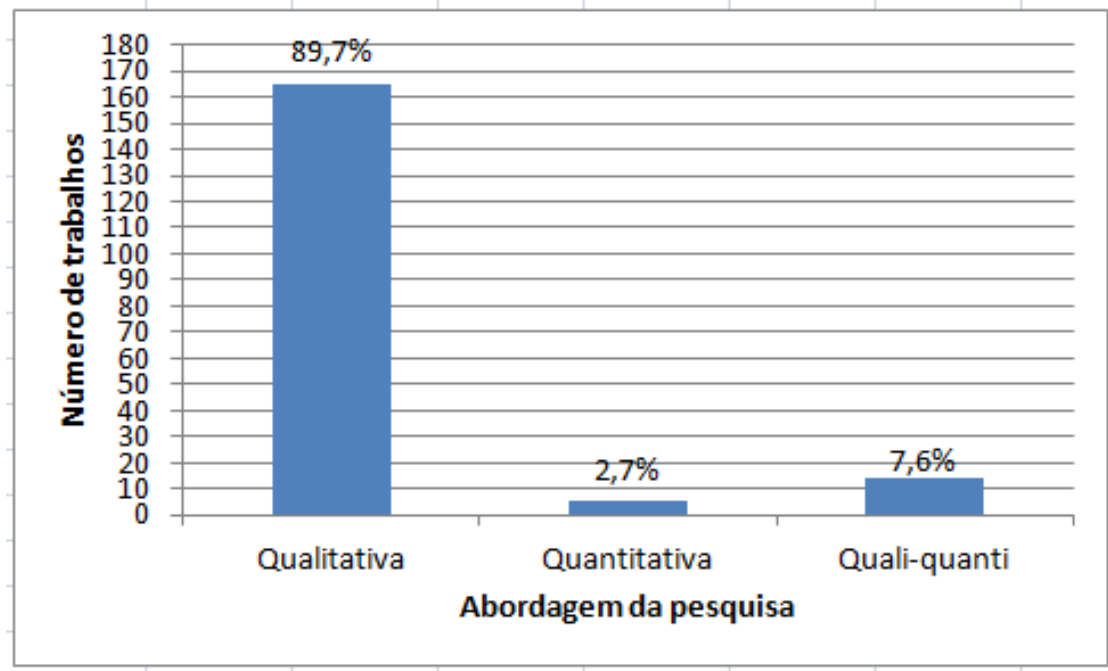

Fonte: Dos autores (2018).

A abordagem qualitativa é comumente utilizada nas Ciências Sociais e tem como característica fundamental a valorização dos aspectos subjetivos e variantes (não absolutos) do problema. Para Saldanha (2010), esses aspectos estão reunidos na ideia de pragmática que prevê, justamente, o estudo do fenômeno ou objeto na forma como se apresenta na realidade, com seus antagonismos e dependência do ponto de vista (relativismo da análise). Nesse sentido, a abordagem pragmática tem sido cada vez mais utilizada nos estudos da OC, Saldanha (2010, p.302-303), diz que,

Chamamos de tradição pragmática a soma de semelhanças de família de abordagens sociológicas [...] e antropológicas[...], soma esta que aqui metodologicamente resulta em um voltar-se para a linguagem, aproximandose de um pragmatismo informacional (no conceito filosófico de pragmatismo, voltado para o antidogmatismo, análise antropológica da linguagem e microestudo de contextos).

Assim, essa aproximação da OC com a pragmática gera pesquisas que só podem ser desenvolvidas a partir de um enfoque qualitativo, pois não têm como objetivo a construção ou constatação de verdades sobre a área, ao invés disso, buscam sua compreensão por meio da contextualização.

O gráfico 3 apresenta a classificação das pesquisas em relação à sua natureza, aplicada, teórica ou teórica-aplicada.

\begin{tabular}{l|l|l|l|l|l|}
\hline (c) RDBCl: Rev. Digit. Bibliotecon. Cienc. Inf. & Campinas, SP & v.17 & $1-16$ & e019018 & 2019 \\
\hline
\end{tabular}




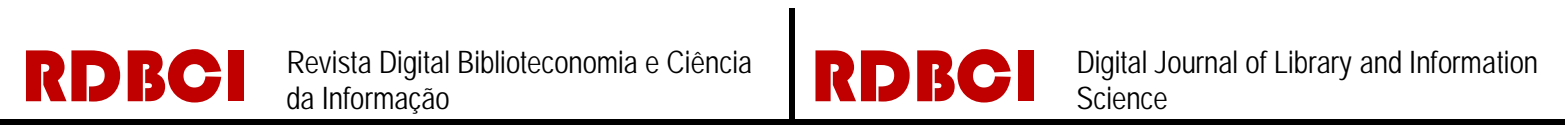

Gráfico 3: Natureza da pesquisa.

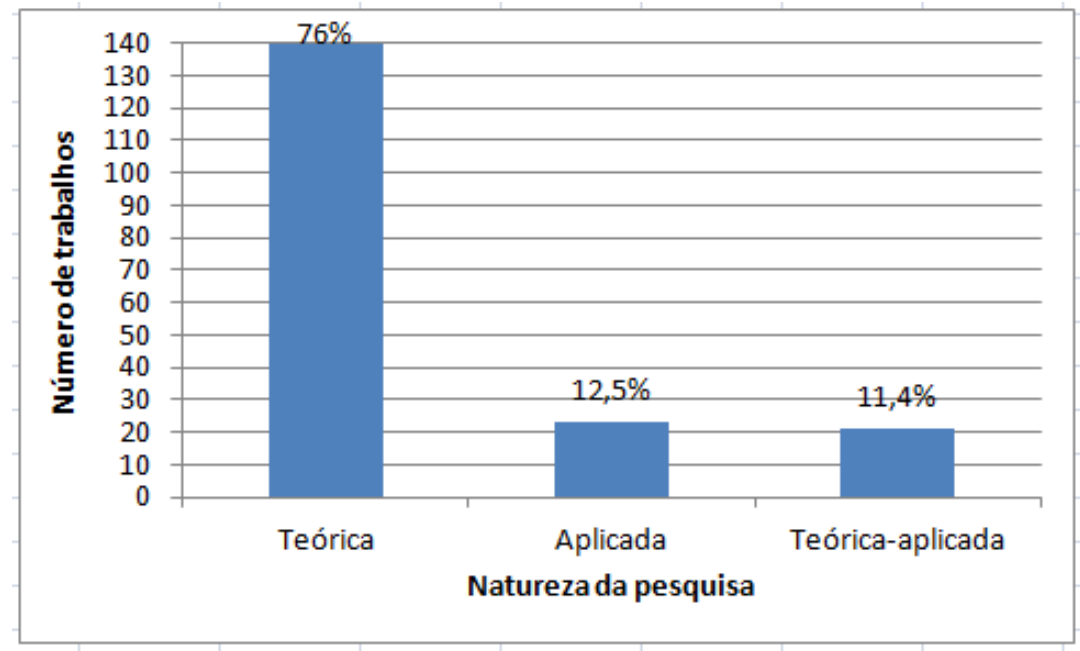

Fonte: Dos autores (2018).

É possível verificar que a maior parte das pesquisas (76\%) do GT2 são teóricas, ou seja, verificam, analisam e/ou constroem teorias. A OC trabalha com conceitos e suas relações, na perspectiva da organização e representação, o que leva a compreensão de que uma abordagem teórica responde melhor a essas características. Mas também aponta para a necessidade das pesquisas serem validadas no campo prático, da experiência. Parece ser um processo de amadurecimento da área, que inicia pela discussão e aprofundamento teórico para se direcionar às constatações práticas.

Os gráficos 4 e 5 apresentam os procedimentos metodológicos adotados nas pesquisas, constituindo o objetivo principal desse trabalho. Dos 184 trabalhos analisados, 82 apresentam mais de um procedimento metodológico, e desses, 4 trabalhos apresentam mais que 2 procedimentos metodológicos, em ambos os casos, diferentes. No processo de análise verificou-se que em 55 pesquisas (30\%) os autores não apresentam de forma clara os procedimentos metodológicos utilizados, apesar de caracterizá-los, em alguns casos. Assim, foi necessário, partindo da análise mais minuciosa dessas pesquisas, nomear o método utilizado. No sentido de exemplificar, citamos o trecho de uma pesquisa analisada que diz que, "O presente trabalho aborda alguns aspectos sobre a leitura de acervos de natureza etnográfica de interesse para a organização e representação da informação. Especificamente, trata das fotografias produzidas nas expedições científicas coordenadas [...]”, os autores não nomearam o procedimento metodológico utilizado, mas, pela caracterização, classificou-se como análise documental, já que são analisadas e representadas as informações de fotografias produzidas em um projeto específico. Outra pesquisa que também não cita explicitamente os procedimentos metodológicos, afirma que, "Discutimos as questões metodológicas relacionadas à organização e representação da informação na Musicologia e na Organização do Conhecimento, procurando mostrar pontos convergentes entre as duas áreas [...]”. A discussão foi realizada a partir de

\begin{tabular}{l|l|l|l|l|l|}
\hline (c) RDBCl: Rev. Digit. Bibliotecon. Cienc. Inf. & Campinas, SP & v.17 & $1-16$ & e019018 & 2019 \\
\hline
\end{tabular}


outros estudos sobre a temática, caracterizando-se como uma pesquisa que apresenta como abordagem metodológica o levantamento bibliográfico.

O gráfico 4 apresenta o primeiro ou único procedimento metodológico utilizado nos trabalhos analisados.

Gráfico 4: Primeiro procedimento metodológico utilizado

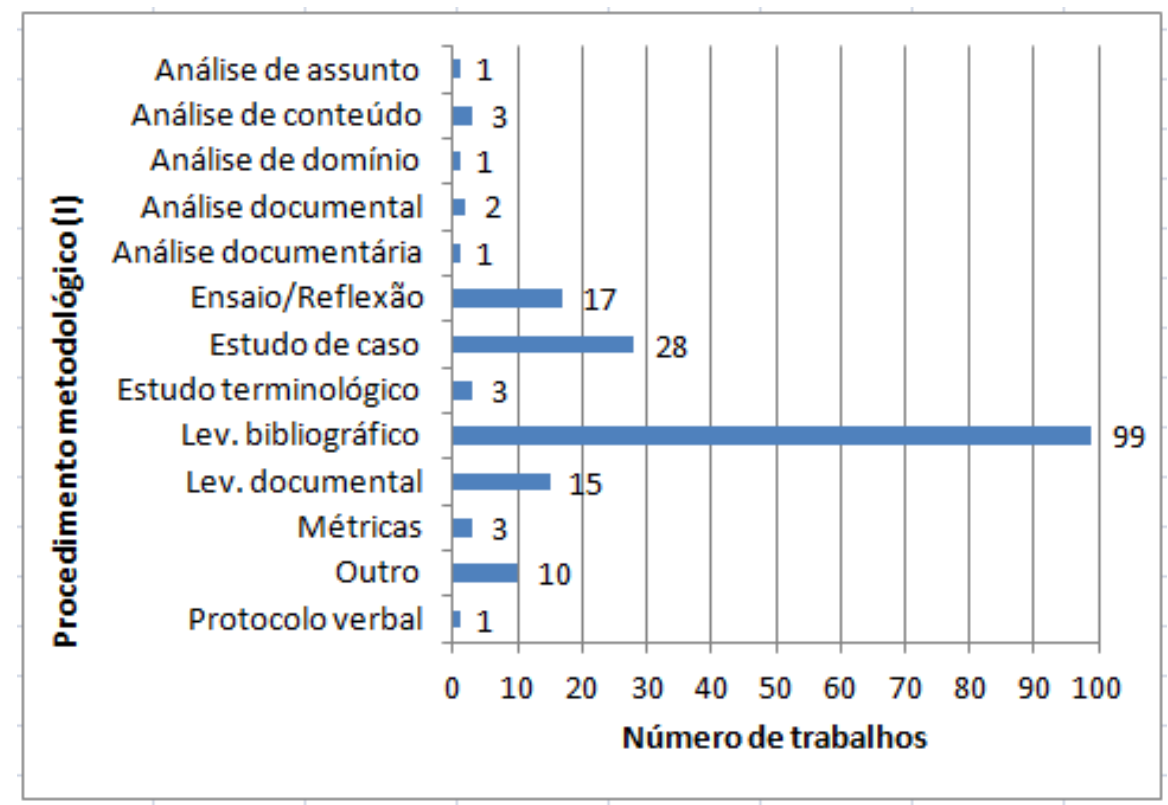

Fonte: Dos autores (2018).

O levantamento bibliográfico foi utilizado em 99 trabalhos (54\%) como procedimento metodológico principal, sendo o método mais apontado. De forma geral, as pesquisas analisadas são qualitativas e utilizam o levantamento bibliográfico como método, assim, podese afirmar que o GT2 desenvolve pesquisas qualitativas, teóricas, e que são fomentadas pela própria literatura científica. Será essa uma característica inerente às pesquisas da área ou temse avançado pouco em relação aos experimentos práticos, pelos motivos já citados? Também chama a atenção o número expressivo de ensaios/reflexões, 17 trabalhos, que não apresentam um aporte metodológico claro, indo de encontro às orientações dadas aos autores que submetem trabalhos ao ENANCIB (2017), que indicam que,

A seção referente aos procedimentos metodológicos deve apresentar de modo claro e sucinto a natureza da pesquisa [qualitativa, quantitativa, qualiquantitativa], o tipo de pesquisa [descritiva-exploratória; documental; bibliográfica; etc.], o método empregado, as técnicas e os procedimentos adotados para a coleta e análise de dados. 
Além desses trabalhos, outros 30\% não seguem a diretriz, não apresentando de forma clara e explícita os procedimentos adotados. Tratando-se de pesquisas científicas, esse fato fragiliza os estudos e pode gerar questionamentos. Também há que se considerar, como dito anteriormente, que algumas pesquisas apresentam características que as tornam de difícil enquadramento metodológico.

Outro resultado que chama a atenção é que apenas um trabalho utiliza a análise de domínio como abordagem metodológica, o que nos leva a questionar o fato de que a abordagem pragmática está mais evidenciada na fundamentação teórica das pesquisas em OC no Brasil do que como uma abordagem metodológica. Guimarães (2014, p.19) aponta que a utilização da análise de domínio como abordagem metodológica nas pesquisas de OC permite uma aproximação mais contextual, afirmando que,

\begin{abstract}
A abordagem da análise de domínio vem sendo especialmente importante para a organização do conhecimento à medida que os processos de tratamento passam a ser abordados a partir do contexto de produção - e de uso - daquele conhecimento, o que vai ao encontro das atuais abordagens culturais da área, que vem sendo objeto de preocupação de pesquisadores como Antônio Garcia Gutierrez, Hope Olson, Caire Beghtol, Grant Campbell, Maria José Lopez Huertas e outros.
\end{abstract}

No entanto, verifica-se que essa abordagem não tem sido utilizada pelos pesquisadores de OC nas pesquisas publicadas no GT2 do ENANCIB. Fato que também pode evidenciar uma carência de pesquisas com abordagens culturais em OC, deixando lacunas nos processos de representação e organização do conhecimento de grupos minoritários, como negros, índios e homossexuais, por exemplo.

Nos 82 trabalhos que utilizaram mais de um procedimento metodológico, o levantamento documental aparece como o segundo método mais citado, utilizado em 19 trabalhos, gráfico 5. Ou seja, o levantamento bibliográfico, método que se baseia em outros estudos é o mais citado como método principal, e é complementado pelo levantamento em fontes primárias, como segundo método mais citado. 


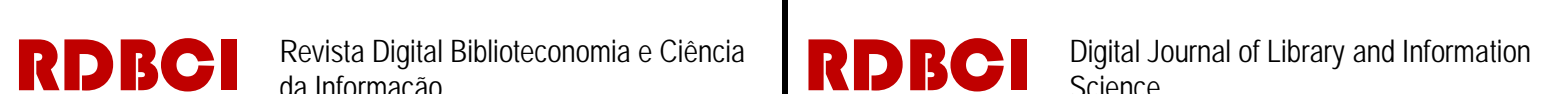

Gráfico 5: Segundo procedimento metodológico utilizado.

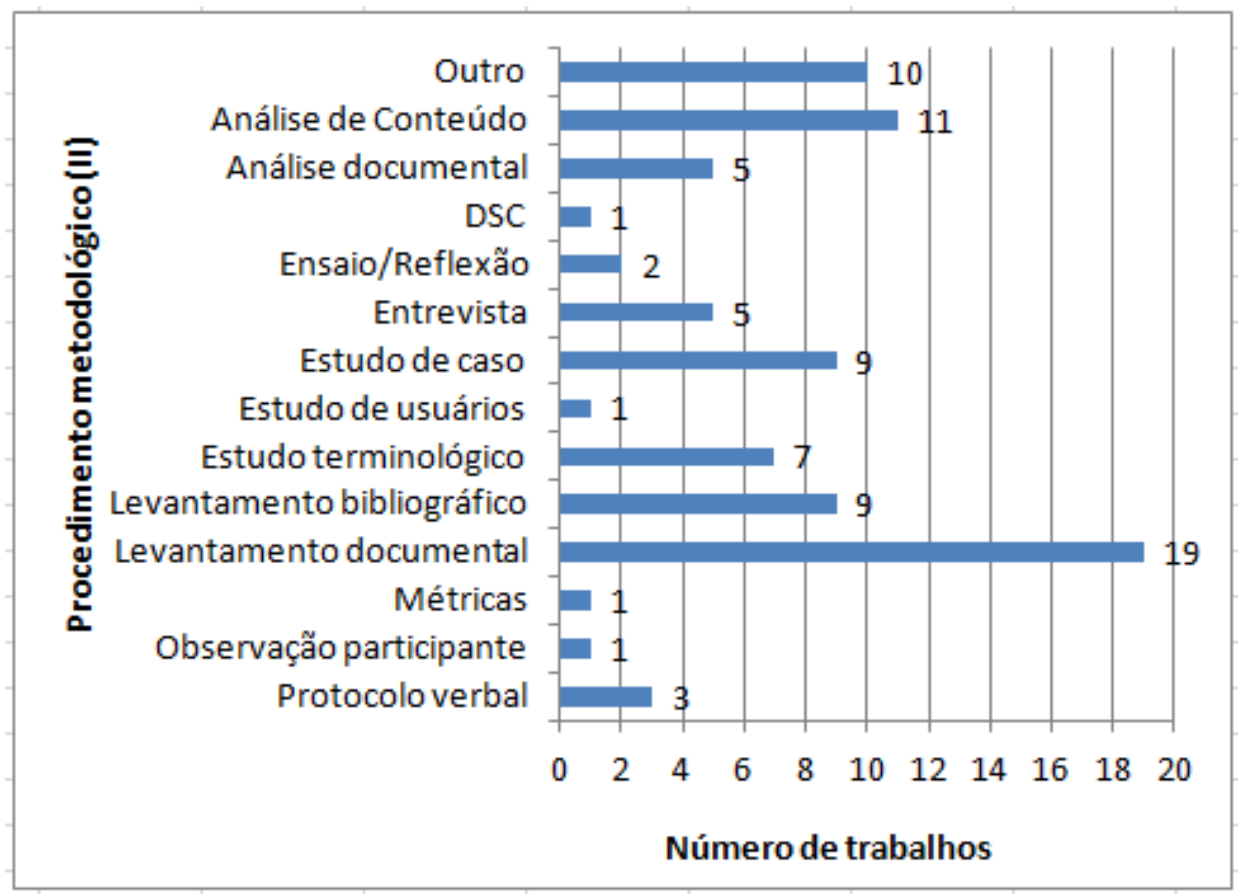

Fonte: Dos autores (2018).

Como dito por Weiss e Bräscher (2014, p.696), “A bibliografia é uma fonte para identificar pontos de vista distintos e construir linguagens de comunicação.” assim, uma pesquisa que se utiliza do levantamento bibliográfico e/ou documental, pode ter um viés pragmático.

Mas, podemos verificar que os segundos métodos citados são bastante pulverizados, além do levantamento documental, a análise de conteúdo, levantamento bibliográfico, estudo terminológico, são utilizados em 11, 9 e 7 trabalhos, respectivamente. Fica evidente que as abordagens metodológicas predominantes são as que têm como corpus de análise a literatura, estando os métodos com viés pragmático, como o protocolo verbal, Discurso do Sujeito Coletivo (DSC) e o estudo de usuários, em menor número.

Os métodos agrupados na categoria 'Outros' não puderam ser definidos e nomeados com exatidão. Para exemplificar, um trabalho que tinha como objetivo propor um procedimento operacional para construção de taxonomias corporativas, afirmava que o procedimento apresentado tinha sido "elaborado por meio de constatações teóricas e práticas vivenciadas pelas autoras, por meio de atividades consultivas”, dificultando o enquadramento metodológico. Ainda, outro trabalho declarou que utilizou "a abordagem metodológica da multimodalidade”, não ficando claro em que consiste essa abordagem. 


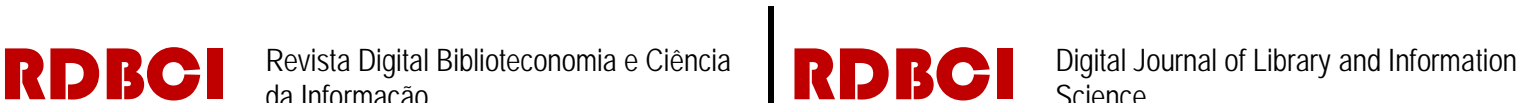

\section{Considerações Finais}

A pergunta que guiou esse estudo, quais procedimentos metodológicos estão sendo utilizados pelas pesquisas em Organização e Representação do Conhecimento no Brasil, ficou um pouco mais clara a partir dos resultados obtidos. Entretanto, como já dito, classificar o método de pesquisa empregado em determinado estudo não é tarefa fácil. As características das pesquisas em uma área diversa como a que investiga o conhecimento, impõem desafios que tornam difícil nomear com exatidão os métodos adotados.

Dos resultados obtidos, ressalta-se o fato de que a maioria dos estudos utiliza o levantamento bibliográfico para viabilizar suas pesquisas. Além de termos um número inexpressivo de pesquisas que se arriscam em abordagens metodológicas não convencionais, que se aproximam da pragmática, como a análise de domínio, discurso do sujeito coletivo ou observação participante, por exemplo. Também as abordagens com um viés mais quantitativo, como as métricas, não tem aparecido nas pesquisas do GT2.

Outro dado relevante é o fato de que 30\% dos trabalhos não declaram os procedimentos metodológicos adotados, o que se deve à dificuldade de enquadrar as características e/ou complexidade da pesquisa em classificações pré-definidas. Por outro lado, essa omissão fragiliza a pesquisa, deixando dúvidas sobre a forma com que o estudo foi conduzido. É possível que a declaração objetiva do método de pesquisa não seja de interesse ou não seja um requisito central para os pesquisadores do GT2, afinal, a abordagem metodológica precisa ser condizente com o contexto em que a área se insere e, neste sentido, classificações ou mesmo métodos limitantes não correspondem ao intenso traço sociológico e pragmático da OC. Porém, a declaração mais detalhada dos procedimentos realizados (independente de uma classificação pré-definida) pode aclarar ao leitor a forma como a pesquisa foi conduzida. Essa possibilidade pode contornar a possível fragilização das pesquisas, como comentamos anteriormente, além de contribuir para melhorar a qualidade da comunicação científica, facilitar a consolidação de resultados entre pesquisadores e, consequentemente, auxiliar no delineamento da própria área de OC. Neste último aspecto vale ressaltar que as abordagens metodológicas são influenciadas e influenciam o desenvolvimento epistemológico da área.Acredita-se que esses resultados fornecem indícios e hipóteses que podem fomentar novas psquisas e permitir um aprofundamento da temática e um olhar mais apurado dos pesquisadores da área sobre as abordagens adotadas.

\section{Referências}

CRESWELL, J. W. Projeto de pesquisa: métodos qualitativo, quantitativo e misto. 2. ed. Porto Alegre: Artmed, 2007. 


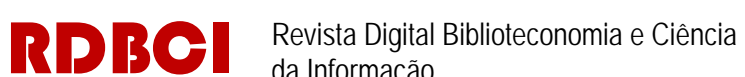 da Informação

ENCONTRO NACIONAL DE PESQUISA EM CIÊNCIA DA INFORMAÇÃO -

ENANCIB, 18., 2017. Disponível em: http://enancib.marilia.unesp.br/index.php/ xviiienancib/ENANCIB. Acesso em 31 jul. 2018.

GIL, A. C. Como elaborar projetos de pesquisa. 5. ed. São Paulo: Atlas, 2010.

GUIMARÃES, J. A. C. Análise de domínio como perspectiva metodológica em organização da informação. Ci. Inf., Brasília, DF, v. 41 n. 1, p.13-21, jan./abr., 2014. Disponível em: http://revista.ibict.br/ciinf/article/view/1415. Acesso em: 31 jul. 2018.

HJØRLAND, B. Theory and metatheory of Information Science: a new interpretation. Journal of Documentation, [London], v. 54, n. 5, p. 606-621, Dec. 1998.

JAPIASSÚ, H.; MARCONDES, D. Dicionário Básico de Filosofia. $4^{\circ}$. ed. atual. Rio de Janeiro: Jorge Zahar. Ed, 2006.

LARROSA, J. O ensaio e a escrita acadêmica. Educação \& Realidade, v. 28, n. 2, p. 101115, 2003. Disponível em: http://www.seer.ufrgs.br/index.php/educacaoerealidade/ article/view/25643/14981. Acesso em: 22 jun. 2018.

LAKATOS, E. M.; MARCONI, M. A. Fundamentos de metodologia científica. 7. ed. São Paulo: Atlas, 2010.

SALDANHA, G. S. Tradições epistemológicas nos estudos de organização dos saberes: uma leitura histórico-epistêmica a partir da filosofia da linguagem. Liinc em Revista, Rio de Janeiro, v. 6, n. 2, p. 300-315, 2010. Disponível em: http://revista.ibict.br/liinc/ article/view/3259. Acesso em: 31 jul. 2018.

SALES, R. O diálogo entre a organização do conhecimento e a ciência da informação na comunidade científica da isko-brasil. In: ENCONTRO NACIONAL DE PESQUISA EM CIÊNCIA DA INFORMAÇÃO, 16., 2015, João Pessoa. Anais... João Pessoa: UFPB, 2015. Disponível em: http://www.brapci.inf.br/index.php/article/view/0000017439/ 57ebfeb65f1964d27bbc53ff108bf22d. Acesso em: 31 jul. 2018.

SMIRAGLIA, R.P. The epistemological dimension of knowledge organization. IRIS-Revista de Informação, Memória e Tecnologia, Fortaleza, v. 2, n. 1, p. 2-11, 2013. Disponível em: https://periodicos.ufpe.br/revistas/IRIS/article/view/498. Acesso em: 22 jun 2018.

VALENTIM, M. L. P. (Org.).Métodos qualitativos de pesquisa em ciência da informação. São Paulo: Polis, 2005.

WEISS, L.C.; BRÄSCHER, M. Pragmática na organização do conhecimento. In: ENCONTRO NACIONAL DE PESQUISA EM CIÊNCIA DA INFORMAÇÃO, 15., 2014, Belo Horizonte. Anais... Belo Horizonte: UFMG, 2014. Disponível em: http://www.brapci.inf.br/index.php/article/view/0000015907/8020b4c345c571fa38cae236f57 4feb3/. Acesso em: 31 jul. 2018. 


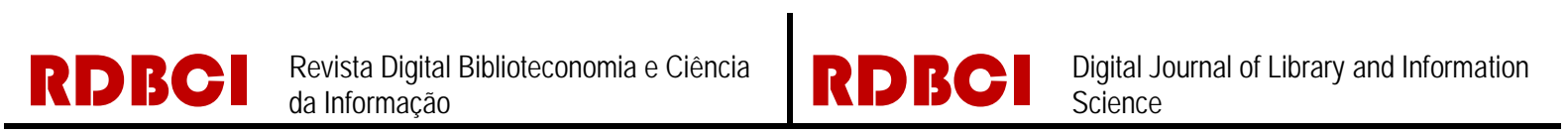

WERSIG, G. Information Science: the study of postmodern knowledge usage. Information Processing \& Management, v. 29, n. 2, 1993. Disponível em:

https://www.sciencedirect.com/science/article/abs/pii/030645739390006Y. Acesso em: 31 jul. 2018. 